\title{
Une approche réductionniste du cancer
}

> L'approche réductionniste attribue l'émergence d'une tumeur et son évolution à des évènements génétiques. L'accumulation d'altérations dans l'expression de gènes provoque l'émergence d'un clone et de sous-clones. Un équilibre dynamique entre les sous-clones et avec leur environnement façonne l'évolution de la tumeur. Sous l'effet d'une pression de sélection protéiforme, une grande hétérogénéité peut apparaître dans la tumeur primitive, entre la tumeur primitive et ses métastases, et entre les métastases. La capacité à identifier cette hétérogénéité dans les tumeurs installées, mais aussi la détection des cellules tumorales avant l'apparition de cette complexité, sont des enjeux de la cancérologie actuelle. <

Dans une approche réductionniste, le cancer est une maladie génique: l'émergence d'une tumeur est la conséquence de l'altération de I'ADN d'une cellule isolée qui acquiert, le plus souvent du fait de la mutation d'un gène (mutation activatrice d'un oncogène ou inhibitrice d'un gène suppresseur de tumeur), parfois de l'altération de son expression par un mécanisme épigénétique, un avantage compétitif par rapport aux cellules voisines. La cellule initialement altérée et ses descendantes définissent le clone tumoral. Les tumeurs constituées sont hétérogènes du fait de l'accumulation, au cours du temps, de mutations somatiques dans une partie des cellules, ce qui définit des sous-clones. Certaines de ces mutations participent activement à la progression de la tumeur alors que d'autres (dites passagères ou neutres) n'ont que peu d'impact sur l'évolution tumorale [1]. Des altérations épigénétiques participent à cette évolution. Les caractéristiques initiales de la cellule affectée, la fonction des gènes

Les six articles publiés dans ce numéro de médecine/sciences regroupent des interventions faites dans le cadre d'un colloque intitulé «La recherche sur le cancer : un champ privilégié pour penser les rapports entre hasard, rédutionnisme et holisme », organisé le 7 novembre 2013, ENS-Paris, 45, rue d’Ulm, 75005 Paris, par le groupe de travail HAREDHOL (hasard, réductionnisme, holisme).

\section{Éric Solary}

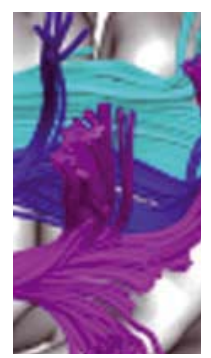

Inserm UMR1009,

Institut Gustave Roussy,

114 , rue Édouard Vaillant, 94805 Villejuif Cedex, France ; université Paris-Sud, faculté de médecine, Le Kremlin Bicêtre, France.

eric.solary@gustaveroussy.fr

altérés, l'environnement tissulaire et la pression thérapeutique vont sculpter l'architecture moléculaire du clone au cours du temps.

Le célèbre schéma proposé par Douglas Hanahan et Robert Weinberg dans Cell en 2000, et actualisé dix ans plus tard [2], résume les propriétés que la cellule transformée acquiert lorsque s'accumulent les altérations génétiques et épigénétiques : activation constitutive de signaux de prolifération, neutralisation de régulateurs négatifs du cycle cellulaire, perte de sensibilité aux signaux de mort, modifications du métabolisme énergétique, instabilité génétique, échappement à la réponse immunitaire, capacité à promouvoir une néoangiogenèse ou une réaction inflammatoire. Les altérations moléculaires qui confèrent à la cellule tumorale une ou plusieurs de ces propriétés sont présentées comme des cibles thérapeutiques potentielles. Cette approche est à la base du concept de médecine dite «personnalisée » (médecine de précision, médecine moléculaire): identifions les altérations des cellules qui composent la tumeur et ciblons celles qui peuvent l'être, avec les médicaments adaptés. Ce concept a permis d'observer des effets thérapeutiques spectaculaires dans certains cancers. La guérison, c'est-à-dire l'éradication complète du clone tumoral, n'est que rarement obtenue.

Dans cette approche réductionniste, le réseau constitué par les gènes transcrits et leurs régulateurs est déterminant dans l'apparition et l'évolution de la tumeur. En d'autres termes, le génotype est responsable du phénotype. La tumeur est complexe, les relations génotype/ phénotype n'étant pas linéaires, et elle est instable et plastique, s'adaptant à la pression évolutive des interactions entre sous-clones et avec l'environnement.

\section{La multiplicité des altérations génétiques}

Il est possible d'identifier au moins une altération génétique ou épigénétique somatique dans la presque totalité des cancers [3]. Le nombre 
d'altérations identifiées dans les cellules tumorales varie énormément d'un type tumoral à un autre, d'un patient à un autre, et parfois d'une partie de la tumeur à une autre.

Si l'on considère les séquences codantes du génome, une tumeur peut être la conséquence de l'altération d'un seul gène, comme on l'observe dans certaines tumeurs pédiatriques. Dans la leucémie myélomonocytaire chronique juvénile par exemple, il n'existe le plus souvent qu'une seule mutation induisant l'activation constitutive d'une voie de signalisation, en l'occurrence la voie Ras [4]. À l'opposé, dans les tumeurs induites par une exposition chronique à des agents mutagènes (mélanomes dus aux ultra-violets, cancers du poumon dus au tabac), on détecte plusieurs centaines de mutations géniques par cellule tumorale $[5,6]$. En dépit des progrès spectaculaires de ces dernières années, l'identification des mutations récurrentes caractérisant un type tumoral donné est encore incomplète, même pour les tumeurs les plus fréquentes. Un calcul récent suggère qu'il faudra séquencer entre 600 et 5000 tumeurs d'un type donné pour avoir une image complète de ses caractéristiques génétiques [7].

Le séquençage du génome complet de plus de 7000 tumeurs issus de 30 organes différents a permis récemment de confirmer cette très grande hétérogénéité et de définir 96 signatures mutationnelles correspondant à des processus oncogéniques distincts [6]. Ces signatures distinguent par exemple les cancers associés au vieillissement de ceux dus à une prédisposition génétique ou à une exposition toxique. La contribution d'une signature mutationnelle à un groupe de tumeurs défini, par exemple, par la localisation de la tumeur primitive, est hétérogène: les tumeurs d'un organe donné n'ont pas toutes la même signature et la répartition des signatures diffère au sein des tumeurs de divers organes. À cette accumulation d'altérations géniques individuelles s'ajoutent des évènements génétiques catastrophiques qui affectent de larges segments du génome: perte de fragments chromosomiques ou de chromosomes entiers, perte d'hétérozygotie sur de larges fragments du génome, tempête mutationnelle au voisinage d'un réarrangement chromosomique [8], cassures chromosomiques brutales suivies d'un réarrangement aléatoire des fragments (chromotripsis) [9], sont autant de composants de la complexité des altérations génétiques qui permettent à la tumeur d'évoluer et de s'adapter, par sélection positive des altérations qui lui sont bénéfiques, dans un environnement tissulaire donné. La notion d'instabilité génétique, souvent associée au cancer, n'est pas constante. II est vrai que certaines tumeurs, par exemple des leucémies et des cancers coliques, apparaissent dans le contexte d'une instabilité génétique due à une altération génétique constitutionnelle ou acquise. Dans d'autres tumeurs, incluant certaines leucémies et certains cancers coliques, le taux de mutations ne semble pas plus élevé dans les cellules tumorales que dans les cellules normales. L'instabilité génomique n'est donc que l'une des composantes de l'évolution génétique des tumeurs.

\section{Les interactions entre altérations génétiques}

Les variations génétiques ne surviennent pas dans le but de transformer la cellule mais s'accumulent au hasard. Une tumeur apparaît lorsqu'une altération génétique confère à la cellule un avantage compétitif en termes de survie et de prolifération dans un environnement donné. Cette altération génétique a un effet oncogénique ou «driver». Une altération peut être «neutre » lorsqu'elle ne confère ni avantage sélectif, ni défaut de compétitivité à la cellule atteinte. Elle est souvent délétère et conduit à l'élimination de la cellule dans laquelle elle survient. Un clone tumoral émerge à la suite d'un nombre élevé de tentatives et d'erreurs, un processus qui se poursuit au cours de la progression tumorale : le temps de doublement des cellules tumorales est très supérieur à celui de la tumeur, indiquant que la majorité des cellules du clone meurent rapidement, sélectionnant les cellules les mieux adaptées à l'environnement dans lequel elles se trouvent.

La croissance tumorale est grossièrement de type Gompertzienne [10]. Pendant plusieurs années, les évènements génétiques qui confèrent à la cellule un avantage compétitif sont neutralisés par des évènements délétères. Lorsque la tumeur a émergé, son développement est limité par diverses contraintes, notamment d'espace et de ressources. Dans le cancer du pancréas par exemple, il faudrait plus de 10 ans avant que le clone émerge, cinq ou six ans pour que des sous-clones ayant un potentiel métastatique se constituent, et trois années supplémentaires pour que les métastases se développent. C'est généralement à ce stade que le diagnostic est fait, l'espérance de vie du patient est alors très réduite [11].

L'environnement tissulaire peut longtemps contenir l'expansion d'un clone, la contrainte étant levée à l'occasion d'un évènement particulier, par exemple thérapeutique : certaines leucémies induites par l'exposition à un traitement cytotoxique administré pour traiter une tumeur solide semblent correspondre à l'expansion d'un clone préexistant, ayant acquis une mutation du gène TP53 au cours du vieillissement normal, le traitement ayant eu pour effet de relâcher le contrôle de l'environnement sur cette cellule mutée [12].

Le caractère bénéfique, neutre, ou délétère d'une altération génétique dépend de plusieurs paramètres. Certains sont propres à la cellule dans laquelle cette altération survient: une mutation peut être neutre lorsque le reste du génome est normal, ou «driver» lorsqu'elle survient dans le contexte de l'altération d'un ou plusieurs autres gènes. Ce phénomène d'épistasie semble avoir joué un rôle important dans l'évolution indépendamment du cancer [13]. Revenons à l'exemple du paragraphe précédent : la mutation de TP53 pourrait permettre à des altérations génétiques supplémentaires induites par la chimiothérapie de générer l'émergence d'un clone leucémique alors que ces mêmes altérations seraient délétères en l'absence de mutation de TP53. 
D'autres sont liés à l'environnement de la cellule mutée, incluant des paramètres physiques de densité cellulaire et d'accessibilité aux ressources nécessaires au métabolisme cellulaire.

La relation génotype/phénotype est complexe et non linéaire. Elle est relativement facile à déchiffrer dans les maladies les plus simples: dans les néoplasmes myéloprolifératifs, la mutation V617F de la tyrosine kinase JAK2 induit une hypersensibilité des progéniteurs hématopoïétiques à certaines cytokines. À l'état homozygote, la mutation confère aux progéniteurs érythroïdes une hypersensibilité à l'érythropoïétine et l'on observe une polyglobulie. Lorsqu'elle est hétérozygote, ce sont les progéniteurs mégacaryocytaires qui sont hypersensibles à la thrombopoïétine et l'on observe une thrombocytose. L'impact de la charge allélique sur le phénotype est alors direct [14]. Dans les tumeurs plus complexes, les interactions entre altérations génétiques compliquent la relation génotype/phénotype. Dans les leucémies aiguës myéloblastiques à caryotype normal, par exemple, les mutations de NPMI (nucleophosmin) sont de bon pronostic lorsqu'elles sont associées à des mutations d'IDHI (isocitrate déshydrogénase) ou $I D H 2$, et de mauvais pronostic lorsqu'elles sont associées à des mutations de FLT3 (récepteur Fms-like tyrosine kinase 3) [15].

Les altérations génétiques spécifiques d'un type tumoral donné, par exemple APC (adenomatous polyposis coli) dans le côlon, sont minoritaires (5-10\% des mutations géniques), mais le contexte dans lequel survient une altération génétique est important. La cellule d'origine et les interactions complexes entre altérations génétiques pourraient expliquer pourquoi les inhibiteurs de BRAF V600E, qui induisent des réponses thérapeutiques spectaculaires dans les mélanomes malins métastatiques, ont des effets limités dans les cancers du côlon porteurs de la même mutation [16].

\section{L’hétérogénéité intratumorale et l'architecture clonale}

Les altérations génétiques qui s'accumulent diversement dans les cellules tumorales génèrent des sous-clones. Certains d'entre eux peuvent avoir un avantage compétitif sur les autres, d'autres pas. Ceux qui ont un avantage compétitif peuvent le perdre à l'occasion d'un nouvel évènement. Les effets de l'accumulation de mutations dans les cellules d'une tumeur donnée ne sont donc pas linéaires, et une tumeur établie est souvent constituée d'un nombre variable de sous-clones dont l'importance relative évolue au cours du temps, naturellement ou sous l'effet de la thérapeutique. Au cours de l'évolution de la tumeur, l'accumulation de mutations n'est pas linéaire, mais de nombreux branchements apparaissent, correspondant à autant de sous-clones.

La pression de sélection de l'environnement peut générer une hétérogénéité intratumorale spectaculaire avec un sous-clone dominant les autres dans une partie de la tumeur tandis qu'un autre domine dans une autre partie de cette même tumeur, les mécanismes générant cette répartition spatiale étonnante restant en grande partie méconnus. Certains sous-clones capables de migrer vers un autre tissu pourraient représenter l'essentiel d'une métastase alors qu'ils ne représentent qu'une petite fraction de la tumeur primitive et la capacité d'un sous-clone à s'installer dans un tissu donné pourrait dépendre de l'environnement tissulaire. Ces observations peuvent avoir un impact considérable en termes de diagnostic, de pronostic et de traitement. L’hétérogénéité intratumorale est, en elle-même, un facteur pronostique péjoratif [17].

La complexité de l'architecture clonale est très variable selon les tumeurs. Dans les pathologies d'évolution lente comme la leucémie myélomonocytaire chronique, les mutations s'accumulent de façon linéaire dans les cellules souches hématopoïétiques. Dans ce compartiment, il est possible de retrouver des cellules représentant les différentes étapes de l'accumulation de ces mutations. Quelques sous-clones émergent du fait de recombinaisons homologues au niveau de certains des gènes mutés, mais ces sous-clones n'évoluent pas pour leur propre compte, ce qui suggère une perte de compétitivité. Au niveau des compartiments plus matures, on ne trouve que les cellules ayant l'ensemble des mutations accumulées dans les cellules souches, suggérant que cette accumulation de mutations confère aux cellules un avantage compétitif lors de la différenciation [18]. Dans les leucémies aiguës lymphoblastiques, la situation est beaucoup plus instable avec de nombreux sous-clones dont plusieurs ont la capacité de reproduire la maladie lorsqu'ils sont injectés à une souris immunodéficiente [19].

Cette dernière observation conduit au concept de cellule souche tumorale ou de cellule capable d'initier et de propager la maladie [20]. Au sein d'une tumeur, le nombre de cellules capables de reproduire la maladie chez l'animal immunodéprimé est très variable. En outre, le phénotype de ces cellules, qui doivent être capables de se renouveler et de reproduire l'évolution sous-clonale de la tumeur [21], est instable et varie avec les altérations génétiques et l'environnement. Ces cellules ont tendance à envahir le clone au cours de l'évolution naturelle de la tumeur, et plus encore sous la pression de sélection des traitements. Le pourcentage de cellules répondant à cette définition tend à augmenter avec l'évolution de la maladie, et, dans certaines tumeurs évoluées, la quasi-totalité des cellules tumorales ont acquis ces propriétés. Cette notion de cellule souche tumorale pourrait être importante en thérapeutique : les sous-clones doués de ces propriétés sont théoriquement la cible privilégiée des traitements.

\section{Les liens entre l’hétérogénéité intratumorale et le traitement}

Le traitement affecte l'architecture de la tumeur en réduisant le plus souvent l'hétérogénéité intratumorale. Il élimine les sous-clones sensibles, réduit la masse 
tumorale avec un bénéfice apparent important. II sélectionne en même temps les clones les plus agressifs et les plus résistants. Dans la leucémie myéloïde chronique, par exemple, l'imatinib sélectionne des sous-clones portant la mutation T351l de BCR-ABL qui confère une résistance au médicament [22], et ce mode de sélection pourrait être particulièrement pertinent dans le contexte des thérapeutiques ciblées. Dans la leucémie lymphoïde chronique cependant, le traitement a un tout autre effet : il permet à de multiples sous-clones de s'étendre et accroît paradoxalement l'hétérogénéité apparente de la tumeur [17]. Le traitement pourrait donc perturber l'équilibre interne d'une tumeur dans laquelle les clones les moins agressifs contrôlent la croissance de clones plus agressifs. La sélection ou l'émergence de sous-clones résistants après traitement peuvent relever aussi de paramètres environnementaux, par exemple la localisation de cellules dans des zones d'hypoxie faiblement vascularisées, de paramètres liés à la cellule d'origine, tels que la résistance intrinsèque des cellules souches d'un tissu, de facteurs épigénétiques, ou de la mise en quiescence de certaines cellules du clone.

Si cette approche réductionniste du cancer doit guider les choix thérapeutiques, il devient important d'analyser l'hétérogénéité tumorale de manière dynamique et fonctionnelle [23]. Les outils nécessaires à cette analyse de l'évolution dynamique des tumeurs, par exemple l'examen des cellules tumorales circulantes [24] ou de I'ADN tumoral circulant [25], sont en développement. II s'agit d'anticiper la sélection de clones résistants, d'imaginer des stratégies permettant l'élimination des sous-clones réfractaires, ou d'exploiter la compétition entre les sous-clones pour stabiliser l'évolution d'une tumeur sans l'éliminer nécessairement. II sera possible aussi de cibler le microenvironnement des sous-clones réfractaires pour moduler leur vascularisation, leurs ressources au sein du tissu ou la réponse immunitaire contre ces sous-clones [26].

Cette vision réductionniste de l'évolution d'une tumeur très plastique dans un environnement instable guide un certain nombre de stratégies thérapeutiques en développement. L'identification précoce des altérations génétiques qui précèdent la complexification de l'architecture clonale, par exemple en s'accumulant avec l'âge [27-29], est un enjeu encore plus important pour tenter de prévenir l'émergence des multiples sous-clones qui apparaissent au cours de l'évolution de la tumeur et de ses métastases et compliquent leur éradication. $\diamond$

\section{SUMMARY}

\section{A reductionnist approach of cancer}

According to a reductionnist view, a malignant tumour emerges and evolves as a consequence of genetic damages that accumulate in a cell. These damages generate a major clone and a number of subclones. A dynamic equilibrium emerges between these sub-clones in a given environment. Upon a multiform selective pressure, a great heterogeneity can appear in the primary tumour, between the primary tumour and its metastases, and between metastases in diverse tissues. The ability to identify this heterogeneity in installed tumours, and the detection of tumour cells before the appearance of this intra-tumour complexity, are major challenges in current oncology. $\diamond$
LIENS D'INTÉRÊT

L'auteur déclare n'avoir aucun lien d'intérêt concernant les données publiées dans cet article.

\section{RÉFÉRENCES}

1. Hanahan D, Weinberg RA. Hallmarks of cancer: the next generation. Cell $2011 ; 144: 646-74$.

2. Bozic I, Antal T, Ohtsuki H, et al. Accumulation of driver and passenger mutations during tumour progression. Proc Natl Acad Sci USA 2010 ; 107 : 18545-50.

3. Versteeg R. Cancer: tumours outside the mutation box. Nature 2014 ; 506 : 438-9.

4. Sakaguchi $\mathrm{H}$, Okuno $\mathrm{Y}$, Muramatsu $\mathrm{H}$, et al. Exome sequencing identifies secondary mutations of SETBPI and JAK3 in juvenile myelomonocytic leukemia. Nat Genet 2013 ; 45 : 937-41.

5. Kandoth C, McLellan MD, Vandin F, et al. Mutational landscape and significance across 12 major cancer types. Nature 2013 ; 502 : 333-9.

6. Alexandrov LB, Nik-Zainal S, Wedge DC, et al. Signatures of mutational processes in human cancer. Nature $2013 ; 500: 415-21$.

7. Lawrence MS, Stojanov P, Mermel CH, et al. Discovery and saturation analysis of cancer genes across 21 tumour types. Nature 2014 ; 505 : 495-501.

8. Nik-Zainal S, Alexandrov LB, Wedge DC, et al. Mutational processes molding the genomes of 21 breast cancers. Cell $2012 ; 149$ : 979-93.

9. Stephens PJ, Greenman CD, Fu B, et al. Massive genomic rearrangement acquired in a single catastrophic event during cancer development. Cell $2011 ; 144: 27-40$.

10. Norton L. A Gompertzian model of human breast cancer growth. Cancer Res $1988 ; 48: 7067-71$.

11. Luebeck EG. Cancer: genomic evolution of metastasis. Nature $2010 ; 467$ : 1053-5.

12. Wong TN, Ramsingh G, Young A, et al. The role of early TP53 mutations on the evolution of therapy-related AML. $55^{\text {th }}$ ASH Annual Meeting, New Orleans, December 7-10, 2013, abstract 5.

13. Breen MS, Kemena C, Vlasov PK, et al. Epistasis as the primary factor in molecular evolution. Nature $2012 ; 490: 535-8$.

14. Vainchenker W, Constantinescu SN. JAK/STAT signaling in hematological malignancies. Oncogene $2013 ; 32$ : 2601-13.

15. Patel JP, Gönen M, Figueroa ME, et al. Prognostic relevance of integrated genetic profiling in acute myeloid leukemia. N Engl J Med 2012 ; 366 : 1079-89.

16. Prahallad A, Sun C, Huang $S$, et al. Unresponsiveness of colon cancer to BRAF(V600E) inhibition through feedback activation of EGFR. Nature 2012 ; $483: 100-3$.

17. Landau DA, Carter SL, Stojanov P, et al. Evolution and impact of subclonal mutations in chronic lymphocytic leukemia. Cell 2013 ; 152 : 714-26.

18. Itzykson R, Kosmider 0 , Renneville $A$, et al. Clonal architecture of chronic myelomonocytic leukemias. Blood $2013 ; 121$ : 2186-98.

19. Anderson K, Lutz C, van Delft FW, et al. Genetic variegation of clonal architecture and propagating cells in leukaemia. Nature $2011 ; 469$ : 356-61.

20. Meacham $C \varepsilon$, Morrison SJ. Tumour heterogeneity and cancer cell plasticity. Nature $2013 ; 501: 328-37$

21. Clappier $\varepsilon$, Gerby B, Sigaux F, et al. Clonal selection in xenografted human $T$ cell acute lymphoblastic leukemia recapitulates gain of malignancy at relapse.J Exp Med 2011 ; 208 : 653-61.

22. Roche-Lestienne C, Soenen-Cornu V, Grardel-Duflos N, et al. Several types of mutations of the Abl gene can be found in chronic myeloid leukemia patients resistant to STI571, and they can pre-exist to the onset of treatment. Blood 2002; 100 : 1014-8.

23. Klco JM, Spencer DH, Miller CA, et al. Functional heterogeneity of genetically defined subclones in acute myeloid leukemia. Cancer Cell $2014 ; 25$ : 379-92.

24. Diaz LA Jr, Bardelli A. Liquid biopsies: genotyping circulating tumor DNA. J Clin Oncol $2014 ; 32$ : 579-86.

25. Krebs MG, Metcalf RL, Carter $L$, et al. Molecular analysis of circulating tumour cells-biology and biomarkers. Nat Rev Clin Oncol 2014 ; 11 : 129-44.

26. Jamal-Hanjani M, Thanopoulou $\varepsilon$, Peggs KS, et al. Tumour heterogeneity and immune-modulation. Curr Opin Pharmacol 2013 ; 13 : 497-503. 


\section{RÉFÉRENCES}

27. Jan M, Snyder TM, Corces-Zimmerman MR, et al. Clonal evolution of preleukemic hematopoietic stem cells precedes human acute myeloid leukemia. Sci Transl Med $2012 ; 4$ : 149ral18.

28. Solary $\varepsilon$, Bernard OA, Tefferi A, et al. The Ten-Eleven Translocation-2 (TET2) gene in hematopoiesis and hematopoietic diseases. Leukemia $2014 ; 28: 485-96$.

29. Jacobs KB, Yeager M, Zhou W, et al. Detectable clonal mosaicism and its relationship to aging and cancer. Nat Genet $2012 ; 44: 651-8$.

\section{Autres articles de ce numéro relatifs au colloque}

- Zielinska A, Issad T. La recherche sur le cancer : un champ privilégié pour penser les rapports entre hasard, réductionnisme et holisme. Med Sci (Paris) $2014 ; 30: 675-8$.

- Morange M. Les modèles explicatifs du cancer : aspects historiques. Med Sci (Paris) 2014 ; $30: 679-82$.

- Sonnenschein C, Soto AM. Le cancer et ses gènes insaisissables. Med Sci (Paris) 2014 ;

$30: 688-92$

- Capp JP. Le rôle des phénomènes aléatoires dans le cancer. Med Sci (Paris) 2014 ; 30 : 693-8. - Kupiec JJ. Comment le hasard intervient-il dans le débat entre holisme et réductionnisme? Conclusion du dossier Cancer/Haredhol. Med Sci (Paris) $2014 ; 30: 699-700$.

\section{POUR EN SAVOIR PLUS (sélection d'articles de $\mathrm{m} / \mathrm{s}$ )}

- Microenvironnements tumoraux : conflictuels et complémentaires. Med Sci (Paris) 2014 ; 30 343-466.

- Fridman WH, Sautès-Fridman C. Le microenvironnement tumoral. Med Sci (Paris) 2014 ; 30 : 359-65.
- Provot S. Contrôle de la croissance et de la dissémination tumorales par le microenvironnement. Med Sci (Paris) 2014 ; 30 : 366-71.

- Hubert S, Abastado JP. Les étapes précoces du processus métastatique. Med Sci (Paris) $2014 ; 30: 378-84$.

- Pellestor F, Gatinois V, Puechberty J, et al. Le chromothripsis. Med Sci

(Paris) $2014 ; 30: 266-73$.

- Le Borgne M, Chartier N, Billaud. Instabilité chromosomique et cancer, enfin des CIN révélateurs. Med Sci (Paris) 2013 ; 29 : 807-10.

- Billaud M. L’hétérogénéité intratumorale. Med Sci (Paris) 2012 ; 28 : 1116-9

- Stoppa-Lyonnet D, Houdayer C. Séquençage de nouvelle génération en génétique médicale. Med Sci (Paris) 2012 ; 28 : 123-4.

- Khuong-Quang DA, Gerges N, Jabado N. Les glioblastomes de l'enfant et du jeune adulte. Une histoire de mutations d'histone et de remodelage de la chromatine. Med Sci (Paris) $2012 ; 28: 809-12$.

- Reynaud C, Billaud M. La théorie de l'équilibre ponctué. Med Sci (Paris) $2011 ; 27: 921-3$

- Selves Y, Olschwang S. Étude des cancers coliques du sujet jeune. Med Sci (Paris) $2009 ; 25: 25-8$.

- Dalle S, Martin-Denavit M, Thomas L. Hypervariabilité génotypique des mélanomes : un défi thérapeutique. Med Sci (Paris) 2006 ; 22 : 178-82.

- Ugo V, James C, Vainchenker W. Une mutation unique de la protéine kinase JAK2 dans la polyglobulie de Vaquez et les syndromes myéloprolifératifs non-LMC. Med Sci (Paris) $2005 ; 21: 669-70$.

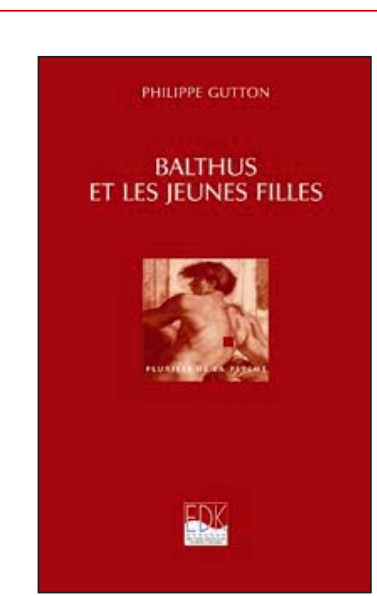

ISBN : 978-2-8425-4190-3 204 pages
À n'en pas douter Balthus (1908-2001) aura été l'un des peintres les plus singuliers du XX' siècle. Les décors étranges où évoluent ses personnages l'ont longtemps inscrit dans la mouvance surréaliste, influence pourtant qu'il rejetait. Les poses suggestives de ses modèles, jeunes filles en fleur, adolescentes à peine pubères, ont incité maints commentateurs à le considérer comme un peintre érotique. Mais là encore Balthus réfutait pareille définition réductrice de son art, lui qui se revendiquant fervent catholique, affirmait : «Le peintre doit être religieux ou n'être pas. »

Philippe Gutton, psychanalyste spécialiste de l'adolescence, reprend la question à ses racines dans un souci de ne pas réduire à une interprétation simplificatrice une création si originale. Quelle énigme recèle cette œuvre ? Que nous disent les figures troublantes qui la hantent? Que révèlent celles-ci de ce temps charnière qu'est l'adolescence? Qu'en tant qu'âge de découverte de la génitalité et de la mort, elle est paradigmatique de la créativité à laquelle tout être humain est tenu de croire s'il veut échapper à la mélancolie ; qu'elle ouvre au mystère de la femme advenante, au secret du vivant, bref qu'interrogeant la puberté féminine en tant qu'elle symbolise l'origine, Balthus, selon la meilleure esthétique, tente et réussit à rendre visible l'invisible de l'humain se faisant.

Philippe GUTTON est psychiatre, psychanalyste. Professeur honoraire des universités, il a fondé en 1983 et dirige depuis la revue Adolescence. Il est l'auteur de nombreux ouvrages, dont, Le pubertaire, Paris, PUF, 1991 ; Violence et adolescence, Paris, In Press, 2002 ; Le génie adolescent, Paris, Odile Jacob, 2008 ; La chambre des amants, Paris, Odile Jacob, 2011.

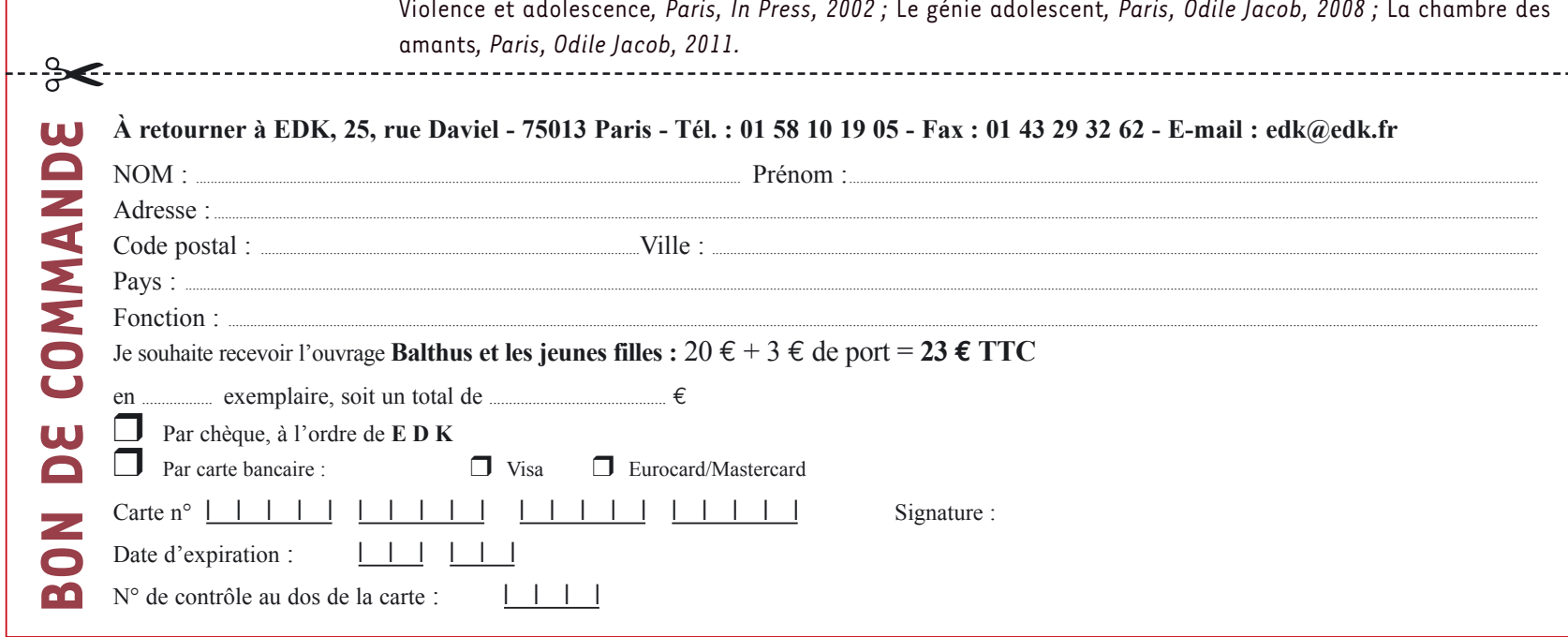

